\title{
WIDE-FIELD IMAGING OF NEARBY GALAXIES IN NEAR-INFRARED BANDS
}

\author{
T. ICHIKAWA ${ }^{1}, K$. TARUSAWA ${ }^{1}, K$. YANAGISAWA ${ }^{2}$, N. ITOH ${ }^{2}$ and M. UENO ${ }^{3}$ \\ ${ }^{1}$ Kiso Observatory, University of Tokyo, Mitake-Mura, Kiso-gun, Nagano 397-01, \\ Japan \\ 2 Department of Astronomy, University of Tokyo, Yayoi, Bunkyo-ku, Tokyo 113, \\ Japan \\ ${ }^{3}$ College of Arts and Science, University of Tokyo, Komaba, Meguro-ku, Tokyo 153, \\ Japan
}

\begin{abstract}
We present the results of wide-field imaging of nearby galaxies observed in the near-infrared using a large format array. The total magnitudes and mass-to-luminosity ratios of NGC 253, M 82, NGC 891 , and some cluster members are discussed.
\end{abstract}

Wide-field imaging with large format arrays is important for studies of the large scale structure of nearby galaxies. At near-infrared wavelengths, galaxies are much less obscured by dust, and the structure of the stellar disk and bulge can be revealed. Moreover, in nearby galaxies a high spatial resolution can be attained and an abundance of data at other wavelengths is available for comparative studies.

The total luminosity of galaxies in near-infrared bands may give more reliable galactic massto-luminosity ratios $(M / L)$ than those obtained in the optical which suffer from dust obscuration. However, the determination of the total magnitude is very sensitive to background subtraction. The variable sky background of $\mathrm{OH}$ emission in near-infrared bands makes mosaicing observations difficult. Registering of adjacent images taken with a small field-of-view is limited if there are only a few stars in the overlapping area. Moreover, spiral galaxy disks follow an exponential law, so a large sky area around a galaxy in an image frame is necessary to determine an accurate background brightness. Observations with a small field-of-view tend to underestimate the galaxy brightness.

To examine the possibility of wide-field imaging in near-infrared bands we made observations with a 512 x 512 PtSi camera (Ueno et al. 1992) attached to the prime focus of the $105 \mathrm{~cm}$ Kiso Schmidt telescope and the newtonian focus of Parkes $25 \mathrm{~cm}$ telescope. The fast $\mathrm{f}$-ratio of the telescopes (F/3.1 and F/3.5) allows observations of the faint part of the galaxies against the bright near-infrared sky background. The field-of-view was $11^{\prime} \times 14^{\prime}$ and $40^{\prime} \times 50^{\prime}$ with a $1.3^{\prime \prime} \times 1.6^{\prime \prime}$ and $6.1^{\prime \prime} \times 9.4^{\prime \prime}$ pixel size, respectively. The resulting image quality and the limiting magnitudes for the Schmidt telescope are reported by Yanagisawa et al. in this volume. Observations were made of NGC 253 in the $K$ band with the $25 \mathrm{~cm}$ telescope and of M 82, N 891, and some other galaxies in $J, H$, and $K$ with the Schmidt telescope.

Figure 1 shows the $K$-band images of NGC 253 and $M$ 82. The amorphous features caused by irregular dust distribution seen in the optical is not clear in the near-infrared images. Instead, 
in the near-infrared M 82 resembles an S0 galaxy with a large bulge and no dust lane in the disk. The bar structure is also evident in the central part, as in NGC 253.

The total magnitudes obtained with the scheme of the RC3 are listed in Table 1 . The analysis for other galaxies is in progress. Galaxies in the Pisces (NGC 295, NGC 452) and Coma clusters (UGC 8017) are also shown for comparison. The masses were obtained with the scheme of Haynes \& Giovanelli (1984), while the mass of M 82 is taken from Sofue et al. (1992). The M/L values in the near-infrared are found to be much smaller than those in the optical.

Table 1. Total magnitude and $M / L$ in near-infrared bands

\begin{tabular}{llcrrrrr}
\hline & Type & r(Mpc) & $J_{\mathbf{T}}$ & $\boldsymbol{H}_{\mathbf{T}}$ & $\boldsymbol{K}_{\mathbf{T}}$ & $\boldsymbol{M} / \boldsymbol{L}_{\boldsymbol{H}}$ & $\boldsymbol{M} / \boldsymbol{L}_{\mathbf{X}}$ \\
\hline \hline NGC 253 & SABc & 3.2 & & & 3.57 & & 1.25 \\
M 82 & SB0 & 3.3 & 5.66 & 4.61 & 4.31 & 0.33 & 0.23 \\
NGC 891 & SAb & $9.5^{1)}$ & 7.26 & 6.29 & 5.76 & 1.83 & 1.19 \\
NGC 295 & SB:b & $75.5^{1)}$ & & 9.50 & & 0.77 & \\
NGC 452 & SBab & $68.7^{1)}$ & & 9.58 & & 1.07 & \\
UGC 8017 & $\ldots$ & $94.2^{1)}$ & & 10.49 & & 1.99 & \\
\hline
\end{tabular}

1) $\mathrm{H}_{\mathrm{o}}=75 \mathrm{~km} / \mathrm{s}^{-1}$
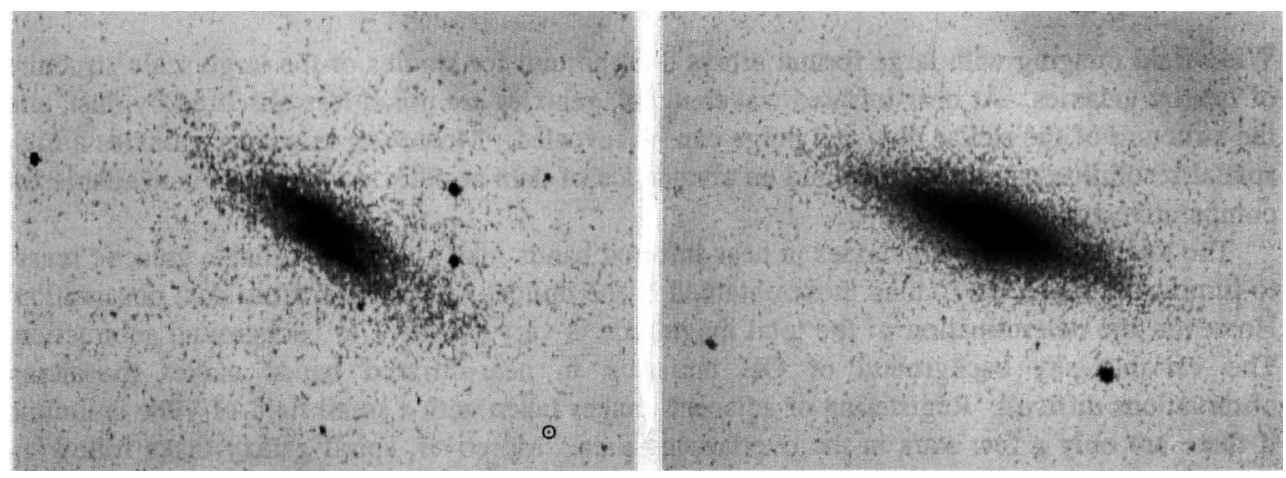

Figure 1. $K$ band images of NGC 253 (left) and M 82 (right).

\section{References}

Haynes and Giovanelli, 1984. Astron. J., 89, 758.

Sofue et al., 1992. Astrophys. J., 395, 126.

Ueno et al., 1992. S.P.I.E., 1762, 423. 